\title{
Ultrahigh energy neutrino interactions and weak-scale string theories
}

\author{
M. Kachelriess \\ TH Division, CERN, CH-1211 Geneva 23, Switzerland \\ M. Plümacher \\ Department of Physics and Astronomy, University of Pennsylvania, Philadelphia, Pennsylvania 19104
}

(Received 22 May 2000; published 16 October 2000)

\begin{abstract}
It has been suggested that ultrahigh energy neutrinos can acquire cross sections approaching hadronic size if the string scale is as low as $1-10 \mathrm{TeV}$. In this case, the vertical air showers observed with energies above the Greisen-Zatsepin-Kuzmin cutoff at $E \approx 6 \times 10^{19} \mathrm{eV}$ could be initiated by neutrinos which are the only known primaries able to travel long distances unimpeded. We have calculated the neutrino-nucleon cross section $\sigma_{N \nu}^{\mathrm{KK}}$ due to the exchange of Kaluza-Klein excitations of the graviton in a field theoretical framework. We have found that $\sigma_{N \nu}^{\mathrm{KK}}$ and the transferred energy per interaction are too small to explain vertical showers even in the most optimistic scenario.
\end{abstract}

PACS number(s): 98.70.Sa, 11.25.Mj, 14.60.Lm

\section{INTRODUCTION}

Several experiments using different techniques have observed ultrahigh energy cosmic rays (UHECR) with energies up to $3 \times 10^{20} \mathrm{eV}[1,2]$. The isotropy of the UHECR arrival directions argues for their extragalactic origin, since galactic and extragalactic magnetic fields cannot isotropize charged particles of such energies. However, all known extragalactic sources of UHECR, such as active galactic nuclei (AGN) [3], topological defects [4] or the local supercluster [5], result in a well pronounced Greisen-Zatsepin-Kuzmin (GZK) cutoff [6] at $E_{\mathrm{GZK}} \approx 6 \times 10^{19} \mathrm{eV}$, although in some cases the cutoff energy is shifted closer to $1 \times 10^{20} \mathrm{eV}$ [5].

As possible solution to this puzzle it has been proposed that the UHE primaries initiating the observed air showers are not protons, nuclei or photons but neutrinos [7-9]. Neutrinos are the only known stable particles which can traverse extragalactic space even at energies $E \gtrsim E_{\mathrm{GZK}}$ without attenuation, thus avoiding the GZK cutoff.

Although only two dozens of UHE events have been observed, we can pin down rather precisely the required interactions of UHE neutrinos. Since the shower profile of Fly's Eyes highest energy event with $E \approx 3 \times 10^{20} \mathrm{eV}$ is well fitted by a proton [10] and also the lateral electron and muon distributions observed by AGASA are consistent with this hypothesis, neutrino-nucleon interactions should mimic nucleon-nucleon interactions at c.m.s. energies $\sqrt{s}$ $\approx 500 \mathrm{TeV}$. In particular, the neutrino-nucleon cross section should reach $\sigma=100-200 \mathrm{mb}$, while the average energy fraction $y$ transferred per interaction to the shower should be large, $y \approx 0.6$.

Most models introducing new physics at a scale $M$ to produce large cross sections for UHE neutrinos fail because experiments generally constrain $M$ to be larger than the weak scale, $M \gtrsim m_{Z}$, and unitarity limits cross sections to be $\mathcal{O}\left(\sigma_{\text {tot }}\right) \leqq 1 / M^{2} \lesssim 1 / m_{Z}^{2}[11]$. String theories with large extra dimensions [12] are different in this respect: if the standard model (SM) particles are confined to the usual $(3+1)$-dimensional space and only gravity propagates in the higher-dimensional space, the compactification radius $R$ of the large extra dimensions can be large, corresponding to a small scale $1 / R$ of new physics. The weakness of gravitational interactions is a consequence of the large compactification radius, since Newton's constant is then given by $G_{N}^{-1}=8 \pi R^{\delta} M_{D}^{\delta+2}$, where $\delta$ is the number of extra dimensions and $M_{D} \sim \mathrm{TeV}$ is the fundamental mass scale. Such a scenario is naturally realized in theories of open strings [13], where SM particles correspond to open strings beginning and ending on $D$-branes, whereas gravitons correspond to closed strings which can propagate in the higher-dimensional space. From a four-dimensional point of view the higherdimensional graviton in these theories appears as an infinite tower of Kaluza-Klein (KK) excitations with mass squared $m_{\vec{n}}^{2}=\vec{n}^{2} / R^{2}$. Since the weakness of the gravitational interaction is partially compensated by the large number of $\mathrm{KK}$ states and cross sections of reactions mediated by spin 2 particles are increasing rapidly with energy, it has been argued in Refs. [8], [9] that neutrinos could initiate the observed vertical showers at the highest energies.

In the calculations of Refs. [9], [14] it was assumed that the massless four-dimensional graviton and its massive KK excitations couple with the usual gravitational strength $\bar{M}_{\mathrm{Pl}}^{-1}=\sqrt{8 \pi} / M_{\mathrm{Pl}}$. Then the sum over all KK contributions to a given scattering amplitude only converges in the case of one extra dimension, and for two or more extra dimensions a cutoff has to be introduced by hand. However, it has recently been pointed out [15] that due to brane fluctuations the effective coupling $g_{\vec{n}}$ of the level $\vec{n} \mathrm{KK}$ mode to fourdimensional fields is suppressed exponentially,

$$
g_{\vec{n}}=\frac{1}{\bar{M}_{\mathrm{P} 1}} \exp \left(-\frac{c m_{\vec{n}}^{2}}{M_{\mathrm{st}}^{2}}\right),
$$

where $c$ is a constant of order 1 or larger, which parametrizes the effects of a finite brane tension [15], and $M_{\mathrm{st}}$ is the string scale. This exponential suppression thereby provides a dynamical cutoff in the sum over all KK modes. We have recalculated the neutrino-nucleon cross section $\sigma_{N \nu}^{\mathrm{KK}}$ due to the 


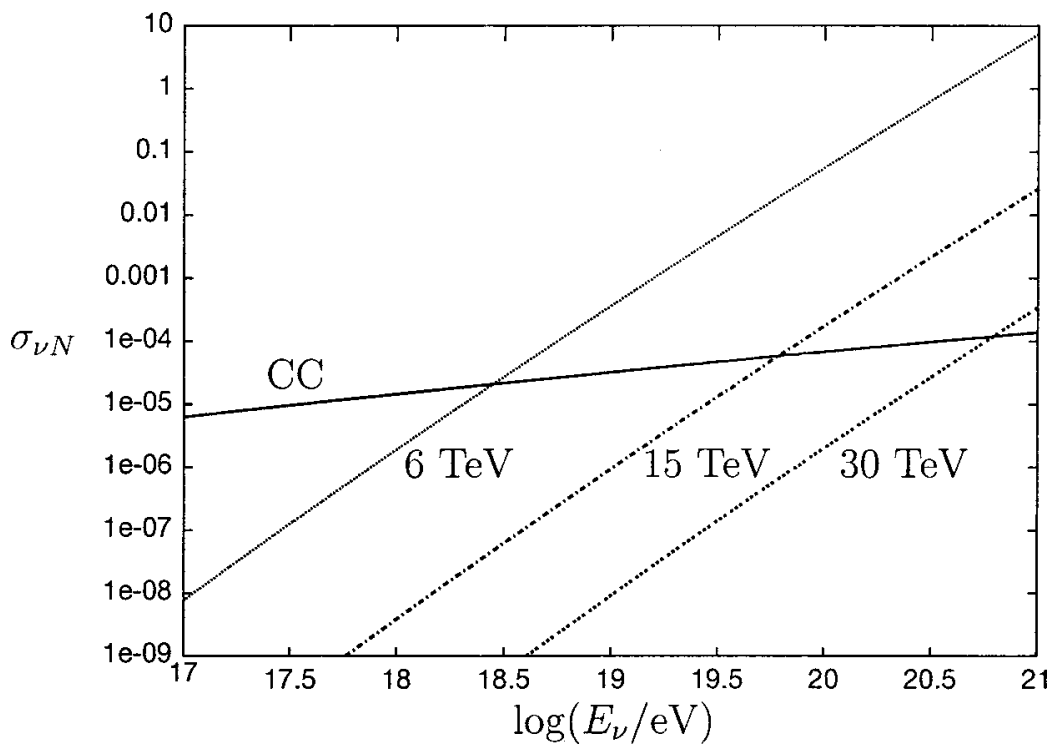

FIG. 1. Neutrino-nucleon cross section $\sigma_{\nu N} / \mathrm{mb}$ due to $W$ exchange (CC) and exchange of KK gravitons as function of $\log \left(E_{\nu} / \mathrm{eV}\right)$ for $M_{\mathrm{st}}=6,15$, and 30 TeV. All for $\delta=2$ and $c=1$. exchange of KK gravitons in a four-dimensional, effective field theory valid for $s \lesssim M_{\mathrm{st}}^{2}$, taking this dynamical cutoff into account. As a consequence, our result for $\sigma_{N \nu}^{\mathrm{KK}}$ is considerably smaller than in previous calculations [14]. Since we have found moreover that the energy transfer per interaction is small at the energies of interest, $y \approx 0.1$, neutrinos behave as deeply penetrating particles and cannot explain the observed vertical air showers. This conclusion holds even if one extrapolates the unitarity violating cross section valid for $s \lesssim M_{\mathrm{st}}^{2}$ to the region $s \gtrsim M_{\mathrm{st}}^{2}$.

We have also derived an upper bound for $\sigma_{N \nu}^{\mathrm{KK}}$ consistent with unitarity for $s \gtrsim M_{\text {st }}^{2}$ using the eikonal method. In this case, the resulting cross section respects the Froissart bound and is numerically too small to lead to observable consequences in UHECR experiments.

\section{NEUTRINO-NUCLEON INTERACTION VIA EXCHANGE OF KALUZA-KLEIN GRAVITONS}

\section{A. Cross section in the low-energy limit $s \ll M_{\text {st }}^{2}$}

At energies below the string scale, effects from KK excitations can be taken into account in an effective fourdimensional field theory. In our calculation we have used the Feynman rules derived in Ref. [16], replacing the KK coupling $\bar{M}_{\mathrm{Pl}}^{-1}$ by the suppressed coupling given in Eq. (1). It is then straightforward to compute the gravitational contributions to partonic cross sections for quark-neutrino scattering

$$
\begin{aligned}
\frac{d \sigma}{d x d \hat{t}}= & \frac{1}{512 \pi \hat{s}^{2}} \frac{P^{2}(\hat{t})}{M_{D}^{4+2 \delta}} \\
& \times\left[32 \hat{s}^{4}+64 \hat{s}^{3} \hat{t}+42 \hat{s}^{2} \hat{t}^{2}+10 \hat{s} \hat{t}^{3}+\hat{t}^{4}\right],
\end{aligned}
$$

and for gluon-neutrino scattering

$$
\frac{d \sigma}{d x d \hat{t}}=\frac{1}{32 \pi \hat{s}^{2}} \frac{P^{2}(\hat{t})}{M_{D}^{4+2 \delta}}\left[2 \hat{s}^{4}+4 \hat{s}^{3} \hat{t}+3 \hat{s}^{2} \hat{t}^{2}+\hat{s} \hat{t}^{3}\right] .
$$

Here we have introduced the Bjorken variable $x$ and $\hat{s}=x s$, $\hat{t}=x t$, where $s=2 m_{N} E_{\nu}$ is the squared c.m.s. energy and $t$ the invariant momentum transfer. The terms in the brackets are symmetric under the exchange $s \leftrightarrow u$ and agree therefore with those of Ref. [9]. The function $P(\hat{t})$ denotes the sum over the propagators of the KK modes, including the couplings from Eq. (1):

$$
P(\hat{t})=R^{-\delta} \sum_{\vec{n}} \frac{\exp \left(-c m_{\vec{n}}^{2} / M_{\mathrm{st}}^{2}\right)}{\hat{t}-m_{\vec{n}}^{2}} .
$$

Due to the small separation $\sim 1 / R$ between the KK levels, the sum can be approximated by an integral and $P(\hat{t})$ is given by

$$
P(\hat{t})=-\pi^{\delta / 2}(-\hat{t})^{\delta / 2-1} \exp \left(-\frac{c \hat{t}}{M_{\mathrm{st}}^{2}}\right) \Gamma\left(1-\frac{\delta}{2},-\frac{c \hat{t}}{M_{\mathrm{st}}^{2}}\right),
$$

where $\Gamma(a, x)$ is the incomplete gamma function as defined, e.g., in Ref. [17]. The $D=4+\delta$ dimensional mass scale $M_{D}$ and the string scale $M_{\mathrm{st}}$ are expected to be of the same order and we have set them equal in the following. Further, in our numerical examples we always consider the case of two extra dimensions, $\delta=2$. However, our results also hold for $\delta$ $>2$.

We have used the CTEQ4 deep inelastic scattering (DIS) [18] parton distribution functions (PDF) to calculate the total nucleon-neutrino cross section. In Fig. 1, the cross sections due to KK exchange are plotted for three different values of $M_{\text {st }}$. For comparison, we show also the charged-current cross section of the SM. We have neglected the neutralcurrent contribution because it is not much larger than the uncertainty of the PDF's. From Fig. 1 it is clear that even for $\sigma_{N \nu}^{\mathrm{KK}}=1-10 \mathrm{mb}$ a value of $M_{\text {st }}$ not much above $1 \mathrm{TeV}$ is required. While present collider experiments do not exclude this possibility, SN 1987A gives $M_{D} \gtrsim 50 \mathrm{TeV}$ [19]. Although the latter limit was obtained for a rather conservative 


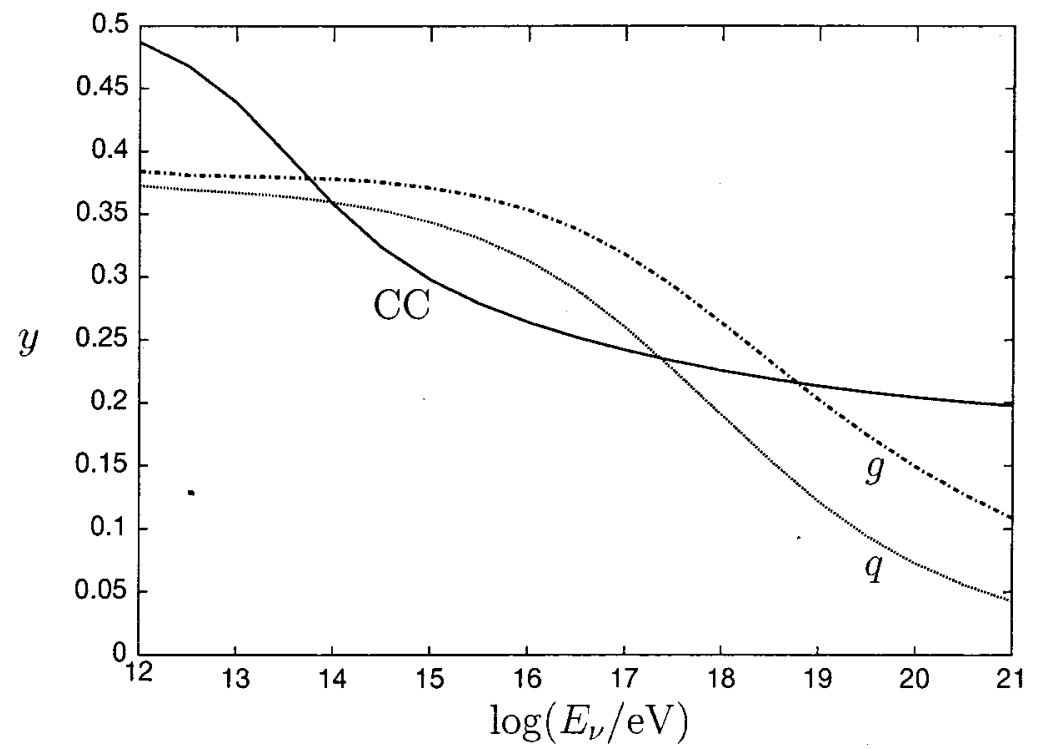

FIG. 2. Energy transfer $y$ in the subreactions with $W$ exchange (CC), exchange of $\mathrm{KK}$ gravitons with quarks $(q)$ and gluons $(g)$ as function of $\log \left(E_{\nu} / \mathrm{eV}\right)$ for $M_{\mathrm{st}}$ $=6 \mathrm{TeV}, \delta=2$ and $c=1$.

choice of supernova parameters, the astrophysical uncertainties inherent in this bound make it plausible that $M_{D}$ $\sim 10 \mathrm{TeV}$ is still compatible with SN 1987A.

The second important quantity characterizing the development of an air shower other than $\sigma_{\text {tot }}$ is the energy transfer $y=\left(E_{\nu}-E_{\nu}^{\prime}\right) / E_{\nu}$. In contrast to charged-current scattering where the electromagnetic shower initiated by the charged lepton is practically indistinguishable from a hadronic shower, only the hit nucleon can initiate an air shower in KK scattering. Therefore, even a neutrino with large $\sigma_{\text {tot }}$ will behave as a penetrating particle if it does not transfer a large fraction of its energy per interaction to the shower.

In Fig. 2, the energy transfer $y$ is shown as function of $E_{\nu}$. At energies of interest, $E_{\nu} \approx 10^{20} \mathrm{eV}$, the transferred energy fraction is only around $y \approx 0.1$, i.e., much smaller than $y \approx 0.6$ typical for nucleon-nucleon collisions.

\section{B. Cross section in the high-energy limit $s \gg M_{\mathrm{st}}^{2}$}

For energies comparable to the string scale $M_{\text {st }}$, the effective theory used above to derive $\sigma_{N \nu}^{\mathrm{KK}}$ breaks down. Since a calculation of $\sigma_{N \nu}^{\mathrm{KK}}$ valid for $s \gg M_{\text {st }}^{2}$ within string theory is beyond the scope of this paper, we restrict ourselves in the following to obtain an upper bound for $\sigma_{N \nu}^{\mathrm{KK}}$. In deriving this bound, we will rely on the assumption that string theory has a better high-energy behavior than four-dimensional field theory. More concretely, we assume that cross sections do not grow faster with $s$ in string theory than allowed by the unitarity bound derived in four-dimensional field theory.

Let us use the Regge language [20,21] to discuss the highenergy behavior of the total cross section $\sigma_{\text {tot }}$. A general Regge amplitude $A_{R}$ can be represented by

$$
A_{R}(s, t)=\beta(t) s^{\alpha(t)},
$$

where the exponent $\alpha(t)$ is given by the relation between spin $\sigma_{i}=\operatorname{int}[\alpha(t)]$ and mass $m_{i}^{2}=t$ of the particles lying on the leading Regge trajectory contributing to the reaction. In our case, the intercept $\alpha(0)$ of this trajectory is equal to the spin of the massless graviton, $\alpha(0)=2$. Since $\sigma_{\text {tot }}$ $\sim s^{-1} \operatorname{Im}\{A(s, 0)\}$, any Regge amplitude with $\alpha(0)>1$ violates both unitarity and the Froissart bound $\sigma_{\text {tot }}(s)<$ const $\times \ln ^{2}\left(s / s_{0}\right)$. In the case of the Pomeron with intercept $\alpha(0)$ $\approx 1.1$, it is well-known that this bad high-energy behavior can only be cured if unitarization produces strong canceling cuts additionally to the Regge poles.

A convenient way to ensure unitarity is the use of the eikonal method. There, the amplitude $A(s, t)$ is given by [21]

$$
A(s, t)=8 \pi s \int_{0}^{\infty} d b b A_{H}(s, b) J_{0}(b \sqrt{-t})
$$

where

$$
A_{H}(s, b)=\frac{e^{i \chi(s, b)}-1}{2 i}
$$

and the eikonal function $\chi(s, b)$ is

$$
\chi(s, b)=\frac{1}{8 \pi s} \int_{-\infty}^{0} d t A_{R}(s, t) J_{0}(b \sqrt{-t}) .
$$

We assume as Ref. [14] that the Regge trajectories are linear, $\alpha(t)=\alpha_{0}+\alpha^{\prime} t$, and that their slope is given by the string tension $\alpha^{\prime}=1 /\left(4 \pi M_{\mathrm{st}}^{2}\right)$. The residue

$$
\beta(t)=-\exp [-i \alpha(t) \pi / 2] e^{a t}
$$

contains the phase of the amplitude and the Reggeon coupling $\propto \exp (a t)$, for which Eq. (1) suggests $a=c / M_{\mathrm{st}}^{2}$. Inserting $A_{R}(s, t)$ into Eq. (9), we obtain 


$$
\begin{aligned}
\chi(s, b) & =-\frac{\left(s / s_{0}\right)^{\alpha_{0}} e^{-i \alpha_{0} \pi / 2}}{8 \pi s d} \exp \left(-\frac{b^{2}}{4 d}\right) \\
& =-\frac{e^{-i \alpha_{0} \pi / 2}}{8 \pi s_{0} d} \exp \left[-\frac{b^{2}}{4 d}+\left(\alpha_{0}-1\right) \ln \left(s / s_{0}\right)\right]
\end{aligned}
$$

where $d=a+\alpha^{\prime}\left[\ln \left(s / s_{0}\right)-i \pi / 2\right]$. If $s \gg s_{0}$ and

$$
b^{2} \gg b_{0}^{2}(s)=4\left(\alpha_{0}-1\right) \alpha^{\prime} \ln ^{2}\left(s / s_{0}\right),
$$

then $\chi(s, b) \approx 0$ and $A_{H}(s, b) \approx 0$. In the opposite case, $b^{2}$ $\ll b_{0}^{2}$, the imaginary part $\operatorname{Im}[\chi(s, b)] \rightarrow \infty$ and $A_{H}(s, b)=i / 2$.

The amplitude

$$
A_{H}(s, b)=\left\{\begin{array}{cc}
i / 2, & b^{2} \ll b_{0}^{2}(s), \\
0, & b^{2} \gg b_{0}^{2}(s)
\end{array}\right.
$$

corresponds to complete absorption on a black disc with radius $b_{0}$. Using $J_{0}(0)=1$ and the optical theorem, the energy dependence of the total cross section follows as

$$
\begin{aligned}
\sigma_{\text {tot }}(s) & =8 \pi \int_{0}^{\infty} d b b \operatorname{Im}\left\{A_{H}(s, b)\right\} \approx 2 \pi b_{0}^{2}(s) \\
& =\text { const } \times \ln ^{2}\left(s / s_{0}\right) .
\end{aligned}
$$

Thus, our result respects the Froissart bound in contrast to the corresponding results of Ref. [14]. The authors of Ref. [14] argued that the asymptotic behavior $\sigma(s) \propto s$ is natural to expect because of the massless graviton. There are two arguments against this interpretation: First, the contribution of any individual KK mode to $\sigma_{\text {tot }}$ is negligible. Therefore, we can omit in the summation over the KK modes the massless graviton, i.e., we can omit the $n=0$ mode in Eq. (4). Then, there is a small, but finite mass gap and the Froissart bound should hold. Second, physical quantities such as total cross sections are infrared finite and although infrared divergences make formally the application of the Froissart bound impossible this should be regarded merely as a technical obstacle.

In Eq. (14), neither the scale $s_{0}$ nor the constant can be fixed within the eikonal method. However, we can obtain an upper bound for $\sigma_{N \nu}^{\mathrm{KK}}$ if we choose the constant as $\sigma_{N \nu}^{\mathrm{KK}}\left(s^{\prime}\right)$ and identify $s^{\prime}$ with the scale above which $s$ wave unitarity is violated. An explicit calculation shows that, as expected, $s^{\prime}$ coincides approximately with $M_{\mathrm{st}}^{2}$. Taking into account that the number of possible targets grows in the nucleon as $\left(s / s^{\prime}\right)^{0.363}[18]$, the total cross section of neutrino-nucleon scattering due to exchange of KK gravitons is bounded by

$$
\sigma_{\text {tot }}(s)=\sigma_{N \nu}^{\mathrm{KK}}\left(M_{\mathrm{st}}^{2}\right) \ln ^{2}\left(s / M_{\mathrm{st}}^{2}\right)\left(s / M_{\mathrm{st}}^{2}\right)^{0.363}, \quad s \gtrsim M_{\mathrm{st}}^{2} .
$$

Finally, we stress that also in the derivation of $\sigma_{\text {tot }}$ in the Regge picture the exponential suppression of high-lying KK modes was essential.

\section{AIR SHOWERS}

First, let us discuss in a very general way how large the total cross section of an UHE primary able to produce the observed vertical air showers should be. The survival probability $N$ at atmospheric depth $X$ of a primary with mean free path $\lambda=m_{\text {air }} / \sigma_{\text {tot }}$ is $N(X)=\exp (-X / \lambda)$, where $m_{\text {air }} \approx 2.4$ $\times 10^{-24} \mathrm{~g}$ is the weight of an "average", air atom. Hence, the probability distribution $p$ of the first interaction point $X_{1}$ has its maximum at $p\left(X_{1}\right)=\lambda$.

For a proton with energy $E=10^{20} \mathrm{eV}$, the mean free path is $\lambda_{p} \approx 40 \mathrm{~g} / \mathrm{cm}^{2}$ and thus a proton air shower is indeed initiated in the top of the atmosphere. After the first interaction, the number of particles in the shower grows until it reaches its maximum at $X_{\max } \approx 800 \mathrm{~g} / \mathrm{cm}^{2}$. Hence, a vertical proton air shower needs almost the complete atmosphere for its development.

How would this picture change for a neutrino with $\lambda_{\nu}$ $=10 \lambda_{p}$, i.e., $\sigma_{\text {tot }}=15 \mathrm{mb}$ ? Taking into account only the delayed start of the shower shifts the shower maximum already $\approx 360 \mathrm{~g} / \mathrm{cm}^{2}$ downwards in the atmosphere. The small energy fraction transferred to the shower per interaction delays the shower development even further. Additionally, the fluctuations of a neutrino shower are enhanced compared to a proton shower. Hence, the shower evolution is clearly different compared to a proton shower. In contrast to Ref. [9], we conclude therefore that even neutrino-nucleon cross sections as large as $15 \mathrm{mb}$ due to $\mathrm{KK}$ exchange are not sufficient to explain vertical air showers by neutrino primaries.

If $\sigma_{N \nu}^{\mathrm{KK}}$ would be considerably larger than $15 \mathrm{mb}$, in principle a detailed simulation of the neutrino air shower development would be necessary. Such issues were studied in the case of glueballinos $\widetilde{G}$ [22], where it was shown that $\widetilde{G}$ showers are clearly distinguishable from proton showers even for $\sigma_{N \tilde{G}} \approx 90 \mathrm{mb}$ and $y \approx 0.1$. It is therefore very likely that the small energy transfer is sufficient to differentiate between showers initiated by proton and by neutrinos interacting through KK gravitons.

Let us now discuss briefly the issue of horizontal air showers. The Fly's Eye experiment presented an upper limit for the neutrino flux from the nonobservation of horizontal air showers [23]. This yields a limit on $M_{\mathrm{st}}$ which is not competitive with accelerator bounds at present. The exact sensitivity of future experiments such as AUGER or OWL is hard to estimate due to the unknown UHE neutrino flux. Signatures would be the anomalous energy and zenith angle distribution of the neutrino showers.

Finally, we address the question if weak-scale string theories can offer additional signatures for UHE neutrino detection. Gauge bosons and higgses could have KK towers of excitations similar to the graviton [24]. In this case, the KK excitations of the $W^{ \pm}$boson with mass $m_{n}^{2}=m_{W}^{2}+\vec{n}^{2} / R^{2}$ result in a corresponding tower of Glashow resonances $\bar{\nu}_{e}$ $+e^{-} \rightarrow W_{n}^{-} \rightarrow$ all. Experimental constraints from existing 
colliders limit the size of the extra dimensions to be $R^{-1}$ $\gtrsim 1 \mathrm{TeV}$ [19]. Therefore, even the first KK resonance at $E_{\mathrm{res}} \approx 1 /\left(2 m_{e} R^{2}\right) \approx 10^{18} \mathrm{eV}\left(R^{-1} / \mathrm{TeV}\right)^{2}$ has a cross section too small to be distinguishable from the SM background. Note also that the couplings of KK excitations with $n>1$ are again exponentially suppressed [25]. Alternative suggestions such as $s$-channel exchange of leptoquarks [26] or squarks in supersymmetric models with $R$-parity violation [27] also fail to generate cross sections of the required magnitude [11], even if possibly existing KK excitations of these states are taken into account.

\section{CONCLUSION}

We have calculated the neutrino-nucleon cross section $\sigma_{N \nu}^{\mathrm{KK}}$ due to the exchange of KK excitations of the graviton taking into account the exponential suppression of modes with $m_{\vec{n}}^{2} \gtrsim M_{\mathrm{st}}^{2}$. Because of the smallness of the resulting cross section and energy transfer per interaction, the neutrino behaves also in these theories as a deeply penetrating particle. In the case that the cross section $\sigma_{N \nu}^{\mathrm{KK}} \propto s^{2}$ continues to grow for $s \gtrsim M_{\text {st }}^{2}$, thereby violating four-dimensional unitarity, future UHECR experiments such as AUGER or OWL could be more sensitive to large extra dimensions than the CERN Large Hadron Collider (LHC). However, an accurate determination of the sensitivity of these experiments would require a string-theoretical calculation of the neutrinonucleon cross section.

\section{ACKNOWLEDGMENTS}

We would like to thank Karim Benakli and Sergey Ostapchenko for helpful comments and the Max Planck Institut für Physik, where this work was started, for hospitality. M.P. was supported in part by the U.S. Department of Energy Grant No. EY-76-02-3071 and in part by the Feodor Lynen Program of the Alexander von Humboldt Foundation.
[1] For recent general reviews of UHECR physics see S. Yoshida and H. Dai, J. Phys. G 24, 905 (1998); V. Berezinsky, Nucl. Phys. B (Proc. Suppl.) 70, 419 (1999); P. Bhattacharjee and G. Sigl, Phys. Rep. 327, 109 (2000).

[2] D. J. Bird et al., Phys. Rev. Lett. 71, 3401 (1993); Astrophys. J. 424, 491 (1995); 441, 144 (1995); N. Hayashida et al., Phys. Rev. Lett. 73, 3491 (1994); M. Takeda et al., ibid. 81, 1163 (1998). For a review of the older experiments see the first citation of Ref. [1] and the literature cited therein. For preliminary results of the HiRes experiment see Fly's Eye Collaboration, J. N. Matthews and C. C. Jui, Nucl. Phys. B (Proc. Suppl.) 87, 411 (2000).

[3] P. L. Biermann and P. A. Strittmatter, Astrophys. J. 322, 643 (1987)

[4] C. T. Hill, D. N. Schramm, and T. P. Walker, Phys. Rev. D 36, 1007 (1987); P. Bhattacharjee, C. T. Hill, and D. N. Schramm, Phys. Rev. Lett. 69, 567 (1992); G. Sigl, astro-ph/9611190; V. Berezinsky and A. Vilenkin, Phys. Rev. Lett. 79, 5202 (1997), and references therein.

[5] V. S. Berezinsky and S. I. Grigorieva, in Proceedings of 16th ICRC, Kyoto, 1979, Vol. 2, p. 8.

[6] K. Greisen, Phys. Rev. Lett. 16, 748 (1966); G. T. Zatsepin and V. A. Kuzmin, JETP Lett. 4, 78 (1966).

[7] V. S. Berezinsky and G. T. Zatsepin, Phys. Lett. 28B, 423 (1969); G. Domokos and S. Nussinov, Phys. Lett. B 187, 372 (1987); J. Bordes, H. Chan, J. Faridani, J. Pfaudler, and S. T. Tsou, Astropart. Phys. 8, 135 (1998).

[8] G. Domokos and S. Kovesi-Domokos, Phys. Rev. Lett. 82, 1366 (1998).

[9] P. Jain, D. W. McKay, S. Panda, and J. P. Ralston, Phys. Lett. B 484, 267 (2000).

[10] F. Halzen, R. A. Vazquez, T. Stanev, and H. P. Vankov, Astropart. Phys. 3, 151 (1995); N. N. Kalmykov, S. S. Ostapchenko, and A. I. Pavlov, Nucl. Phys. B (Proc. Suppl.) 52B, 17 (1997)
[11] G. Burdman, F. Halzen, and R. Gandhi, Phys. Lett. B 417, 107 (1998).

[12] N. Arkani-Hamed, S. Dimopoulos, and G. Dvali, Phys. Lett. B 429, 263 (1998); I. Antoniadis, N. Arkani-Hamed, S. Dimopoulos, and G. Dvali, ibid. 436, 257 (1998); N. ArkaniHamed, S. Dimopoulos, and G. Dvali, Phys. Rev. D 59, 086004 (1999).

[13] C. Angelantonj, M. Bianchi, G. Pradisi, A. Sagnotti, and Y. S. Stanev, Phys. Lett. B 385, 96 (1996); M. Berkooz and R. G. Leigh, Nucl. Phys. B483, 187 (1997); G. Zwart, ibid. B526, 378 (1998); Z. Kakushadze, ibid. B512, 221 (1998); Z. Kakushadze and G. Shiu, Phys. Rev. D 56, 3686 (1997); Nucl. Phys. B520, 75 (1998); L. E. Ibáñez, J. High Energy Phys. 07, 002 (1998); D. O’Driscoll, hep-th/9801114; G. Aldazabal, A. Font, L. E. Ibáñez, and G. Violero, Nucl. Phys. B536, 29 (1999); L. E. Ibáñez, C. Muñoz, and S. Rigolin, ibid. B553, 43 (1999); Z. Kakushadze and S. H. Henry Tye, Phys. Rev. D 58, 126001 (1998); G. Shiu and S. H. Henry Tye, ibid. 58, 106007 (1998); Z. Kakushadze, Phys. Lett. B 434, 269 (1998); Phys. Rev. D 58, 101901 (1998); Nucl. Phys. B535, 311 (1998); M. Cvetič, M. Plümacher, and J. Wang, J. High Energy Phys. 04, 004 (2000); G. Aldazabal, L. E. Ibáñez, F. Quevedo, and A. M. Uranga, ibid. 08, 002 (2000).

[14] S. Nussinov and R. Shrock, Phys. Rev. D 59, 105002 (1999).

[15] M. Bando, T. Kugo, T. Noguchi, and K. Yoshioka, Phys. Rev. Lett. 83, 3601 (1999); J. Hisano and N. Okada, Phys. Rev. D 61, 106003 (2000).

[16] G. F. Giudice, R. Rattazzi, and J. D. Wells, Nucl. Phys. B544, 3 (1999); T. Han, J. D. Lykken, and R. Zhang, Phys. Rev. D 59, 105006 (1999).

[17] A. Erdelyi, Higher Transcendental Functions (McGraw-Hill, New York, 1953).

[18] H. L. Lai et al., Phys. Rev. D 55, 1280 (1997). 
[19] For a review of experimental bounds see M. E. Peskin, hep-ph/0002041; I. Antoniadis and K. Benakli, hep-ph/0004240.

[20] R. J. Eden, High Energy Collisions of Elementary Particles (Cambridge University Press, Cambridge, England, 1967).

[21] P. D. B. Collins, An Introduction to Regge Theory and High Energy Physics (Cambridge University Press, Cambridge, 1977).

[22] V. Berezinsky, M. Kachelriess, and S. Ostapchenko, Report No. CERN-TH 2000-135.
[23] R. M. Baltrusaitis et al., Phys. Rev. D 31, 2192 (1985).

[24] I. Antoniadis, Phys. Lett. B 246, 377 (1990).

[25] L. Dixon, D. Friedan, E. Martinec, and S. Shenker, Nucl. Phys. B282, 13 (1987); S. Hamidi and C. Vafa, ibid. B279, 465 (1987); I. Antoniadis and K. Benakli, Phys. Lett. B 326, 69 (1994).

[26] R. W. Robinett, Phys. Rev. D 37, 84 (1988); M. A. Doncheski and R. W. Robinett, ibid. 56, 7412 (1997).

[27] M. Carena, D. Choudhury, S. Lola, and C. Quigg, Phys. Rev. D 58, 095003 (1998). 